\title{
On the Space which admits a given Continuous Transformation Group in the extended sense.
}

By

Takasi SibatA.

(Received 20, 4, 1934.)

We shall consider the space in which parallel directions along every curve are unaltered by all the transformations of a given continuous transformation group. This case we call the space which admits the given group in the extended sense.(1)

First, by using the notations of Lie's symbols of infinitesimal transformations, we shall obtain the conditions in which a space admits a group in the extended sense.

Next, we will examine the case when the number of the order of the group admitted by a space in the extended sense, is maximum.

Lastly, in the general space which admits a group in the extended sense, we shall obtain the relations between the covariant derivatives and the transformation-derivatives ${ }^{(2)}$ for a vector field.

1. Let us consider a space $V_{n},{ }^{(3)}$ and let the coordinates be $x^{1}, \ldots, x^{n}$, and the coefficients of connection be $L_{j k}^{i}$. Then we can define the parallelism of vectors in $V_{n}$ by the following infinitesimal transformations :(4)

(1) When the parallelism of vectors in a space, not parallel directions, is unaltered by all the transformations of a given group, I say that the space admits the group (not in the extended sense). I have treated this case in this journal 4 (1934), 111-126.

(2) T. Sibata, this journal 4 (1934), 116-117.

(3) In this paper we shall employ certain notations due to L.P. Eisenhart, NonRiemannian Geometry (1927).

(4) T. Sibata, loc. cit., 112. 


$$
T_{i}=\frac{\partial}{\partial x^{i}}-L_{\alpha i}^{\lambda} \dot{x}^{\alpha} \frac{\partial}{\partial \dot{x}^{\lambda}} \quad(i=1, \ldots, n)
$$

where $\dot{x}^{\lambda}(\lambda=1, \ldots, n)$ are the components of any vector:

Now let an $r$-parameter continuous transformation group be given by the following infinitesimal transformations

$$
S_{k}=\xi_{k}^{i}(x) \frac{\partial}{\partial x^{i}} \quad(k=1, \ldots, r),
$$

and any infinitesimal transformation belonging to this group be

$$
S=\xi^{i} \frac{\partial}{\partial x^{i}}
$$

If we denote the extended infinitesimal transformation of $S$, by

$$
\dot{S}=\xi^{i} \frac{\partial}{\partial x^{i}}+\frac{\partial \xi^{i}}{\partial x^{\alpha}} \dot{x}^{\alpha} \frac{\partial}{\partial \dot{x}^{i}},
$$

then $T_{i}(i=1, \ldots, n)$ are transformed by (3) as follows

$$
T_{i}^{\prime}=T_{i}+t\left(\dot{S} T_{i}\right)+\frac{t^{2}}{2 !}\left(\dot{S}\left(\dot{S} T_{i}\right)\right)+\ldots \quad(i=1, \ldots, n) .
$$

The necessary and sufficient condition that the space whose parallelism of vectors is defined by (1), admits the group $S$ in the extended sense, is that, for all values of $t, T_{i}^{\prime}(i=1, \ldots, n)$ must have the forms

$$
\rho_{i}^{l} T_{l}+\varphi_{i} \dot{x}^{\lambda} \frac{\partial}{\partial \dot{x}^{\lambda}} \quad(i=1, \ldots, n),
$$

where $\rho_{i}^{l}(i, l=1, \ldots, n)$ are certain functions of $x$, and $\varphi_{i}(i=1$, $\ldots, n)$ are arbitrary functions of $x$. Therefore, it must be that

$$
\left(\dot{S} T_{i}\right)=\rho_{i}^{l} T_{l}+\varphi_{i} \dot{x}^{\lambda} \frac{\partial}{\partial \dot{x}^{\lambda}} \quad(i=1, \ldots n) ;
$$

hence $\left(\dot{S}\left(\dot{S} T_{i}\right)\right)$, etc. can be expressed in the form of (5), and therefore $T_{i}^{\prime}(i=1, \ldots, n)$ also. But comparing the coefficients of $\frac{\partial}{\partial x^{l}}$ on both sides of $(6)$, we have

$$
\rho_{i}^{l}=-\frac{\partial \xi^{l}}{\partial x^{i}} .
$$


Hence (6) becomes

$$
\left(\dot{S} T_{i}\right)=-\frac{\partial \xi^{l}}{\partial x^{i}} T_{l}+\varphi_{i} \dot{x}^{\lambda} \frac{\partial}{\partial \dot{x}^{\lambda}} \quad(i=1, \ldots, n),
$$

where $\varphi_{i}(i=1, \ldots, n)$ are arbitrary functions of $x$. So we have the result: The relations (7) are the necessary and sufficient conditions that the space admits the group $S$ in the extended sense.

Further, comparing the coefficients of $\frac{\partial}{\partial \dot{x}^{\lambda}}$ on both sides of (7), we have

$$
\frac{\partial^{2} \xi^{\alpha}}{\partial x^{i} \partial x^{j}}+L_{i k}^{\alpha} \frac{\partial \xi^{k}}{\partial x^{j}}+L_{k j}^{\alpha} \frac{\partial \xi^{k}}{\partial x^{i}}+\xi^{h} \frac{\partial L_{i j}^{\alpha}}{\partial x^{h}}-L_{i j}^{k} \frac{\partial \xi^{\alpha}}{\partial x^{k}}=\delta_{i}^{\alpha} \phi_{j}
$$

Conversely, from (8) we can easily deduce (7). So we have the result: The relations (8) are the necessary and sufficient conditions that the space admits the group $S$ in the extended sense.

If we denote the symmetric and the antisymmetric part of $L_{j k}^{i}$ by $\Gamma_{j k}^{i}$ and $\Omega_{j k}^{i}$ respectively, then the equations (8) are rewritten as follows :

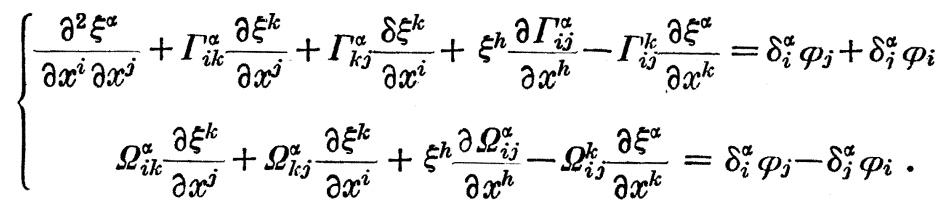

Specially, when $\Omega_{i j}^{\alpha}=0$, namely when the space is symmetric, we have from $(10) \varphi_{i}=0(i=1, \ldots, n)$, and (9) becomes

$$
\frac{\partial^{2} \xi^{\alpha}}{\partial x^{i} \partial x_{j}}+\Gamma_{i k}^{\alpha} \frac{\partial \xi^{k}}{\partial x^{j}}+\Gamma_{k j}^{\alpha} \frac{\partial \xi^{k}}{\partial x^{i}}+\xi^{h} \frac{\partial \Gamma_{i j}^{\alpha}}{\partial x^{h}}-\Gamma_{i j}^{k} \frac{\partial \xi^{\alpha}}{\partial x^{k}}=0
$$

This is not other than the condition that the space, the coefficients of connection being $\Gamma_{i j}^{\alpha}$, admits the group $S$ (not in the extended sense). ${ }^{(1)}$ So we have

Theorem 1. When a symmetric space admits a group in the extended sense, then the space also admits the group (not in the extended sense).

(1) T. Sibata, loc. cit., 113. 
2. We shall next determine the conditions in which the number of the order of the group admitted by a space in the extended sense, becomes maximum. Using the method which Eisenhart adopted in his treatise $e^{(1)}$, we see that this occurs when the Weyl tensor of $\Gamma_{i j}^{\alpha}$ of this space vanishes; and in this case by suitably choosing the coordinate system, $\varphi_{i}$ and $\xi^{i}(i=1, \ldots, n)$, which satisfy (9), become as follows

$$
\begin{aligned}
& \varphi_{i}=a_{i} \\
& \xi^{i}=a_{h} x^{h} x^{i}+b_{l}^{j} x^{l}+c^{i} \quad(i=1, \ldots, n)
\end{aligned}
$$

where $a^{\prime} \mathrm{s}, b^{\prime} \mathrm{s}$, and $c^{\prime} \mathrm{s}$ are arbitrary constants.

Hence, if we choose $a^{\prime} \mathrm{s}, b^{\prime} \mathrm{s}$, and $c^{\prime} \mathrm{s}$ in (12) such that (12) satisfies (10) for a given $\Omega_{i j}^{\alpha}$, for all such $a^{\prime}$ 's, $b$ 's and $c^{\prime}$ 's (12) gives the greatest group which can be admitted by a space in the extended sense.

If we suppose that such $a$ 's, $b$ 's and $c$ 's are all arbitrary constants (12) would give the following $n^{2}+2 n$ infinitesimal transformations

$$
\frac{\partial}{\partial x^{i}}, \quad x^{l} \frac{\partial}{\partial x^{i}}, \quad x^{l} x^{j} \frac{\partial}{\partial x^{j}} \quad(i, l=1, \ldots, n),
$$

i.e. the general projective transformation group. By actual calculation we find that $\xi^{i}$ corresponding to all the infinitesimal transformations of (13) does not satisfy (10) for each value of $\Omega_{i j}^{\alpha}$.

Hence the greatest group which can be admitted by a space in the extended sense, must be a sub-group of (13). From Lie's theorem ${ }^{(2)}$, we know that the greatest sub-group of the general projective group (13) has $n(n+1)$ parameters, and they are similar either to the general linear group $\frac{\partial}{\partial x^{i}}, x^{l} \frac{\partial}{\partial x^{i}}(i, l=1, \ldots, n)$, or to $x^{l} \frac{\partial}{\partial x^{i}}, x^{l} x^{j} \frac{\partial}{\partial x^{j}}(i, l=1$, $\ldots \ldots, n)$. But by actual calculation we see that, for $\xi^{i}$ corresponding to the later group, (8) doesnot hold for each value of $L_{j k}^{i}$, and for $\xi^{i}$ corresponding to the former group, (8) holds when, and only when,

$$
L_{j k}^{i} \stackrel{*}{=} \delta_{j}^{i} \psi_{k}
$$

where $\psi_{k}(k=1, \ldots, n)$ are the components of an arbitrary covariant vector. So we have

Theorem 2. The greatest group which can be admitted by a space in the extended sense, has $n(n+1)$ parameters and is similar to

(1) Eisenhart, loc. cit., 126-131.

(2) S. Lie, Theorie der Transformationsgruppe 1 (1930), 569. 
the general linear group $\frac{\partial}{\partial x^{i}}, x \frac{\partial}{\partial x^{i}}(i, l=1, \ldots, n)$, and in this case the coefficient of connection has the form

$$
L_{j k}^{i} \stackrel{*}{=} \delta_{j}^{i} \psi_{k}
$$

where $\psi_{k}(k=1, \ldots, n)$ expresses an arbitrary covariant vector.

3. Lastly, in the general space which admits the group $S$ in the extended sense, we will obtain the relations between the covariant derivative :

$$
\nabla_{i} v^{\lambda}=\frac{\partial v^{\lambda}}{\partial x^{i}}+\Gamma_{\alpha i}^{\lambda} v^{\alpha}
$$

and the transformation-derivative ${ }^{(1)}$ by $S$ :

$$
\Delta v^{\lambda}=\xi^{\alpha} \frac{\partial v^{\lambda}}{\partial x^{\alpha}}-v^{\alpha} \frac{\partial \xi^{\lambda}}{\partial x^{\alpha}}
$$

for an arbitrary vector field $v^{\lambda}(x)$. We have seen that the space defined by $T_{1}, \ldots, T_{n}$ (see (1)) admits the group $S$ in the extended sense when, and only when,

$$
\left(\dot{S} T_{i}\right)=-\frac{\partial \xi^{l}}{\partial x^{i}} T_{l}+\phi_{i} \dot{x}^{\lambda} \frac{\partial}{\partial \dot{x}^{\lambda}} \quad(i=1, \ldots, n)
$$

If we apply the operators on both sides of this relation, to an arbitrary system of equations of the form

$$
f^{\lambda} \equiv \dot{x}^{\lambda}-v^{\lambda}(x)=0 \quad(\lambda=1, \ldots, n),
$$

and substituting $\dot{x}^{\lambda}=v^{\lambda}(x)(\lambda=1, \ldots, n)$ in the results, we have

$$
\nabla_{i} \Delta v^{\lambda}-\Delta \nabla_{i} v^{\lambda}=\phi_{i} v^{\lambda}
$$

Conversely from (14) we can easily deduce the relations (7). So we have

(1) T. Sibata, loc. cit., 116, 117. 
Theorem 3. A space admits a group in the extended sense when, and only when, for any arbitrary vector field $v^{\lambda}(x)$, the following relations hold

$$
\nabla_{i} \nabla v^{\lambda}-\Delta \nabla_{i} v^{\lambda}=\phi_{i} v^{\lambda}
$$

where $\phi_{i}(i=1, \ldots, n)$ are arbitrary functions of $x$. 